\title{
Quantifying Homicide Injuries: A Swedish Time Trend Study Using the Homicide Injury Scale
}

\author{
Fredrik Tamsen 1,3*, Joakim Sturup ${ }^{2,3}$, Ingemar Thiblin ${ }^{1,3}$
}

\author{
'Department of Surgical Sciences, Uppsala University, Sweden \\ ${ }^{2}$ Karolinska Institute, Stockholm, Sweden \\ ${ }^{3}$ The Swedish National Board of Forensic Medicine \\ *Corresponding Author E-mail: fredriktamsen@gmail.com
}

\begin{abstract}
:
Studies report that the homicide rate has decreased considerably in most Western countries since the 1990s. However, few studies have examined the level of injury in homicides. The injury severity in homicide victims was studied in the Stockholm area using both the Homicide Injury Scale (HIS) and the number of lethal injuries per victim. Cases were included from four periods; 1976-78, 1986-88, 1996-98, and 2006-08. The number of homicides with overkill according to the HIS was significantly higher in 1996-98 compared to 1976-78. Compared with 1976-78, the number of lethal injuries per victim was significantly higher both in 1996-98 and 2006-2008. There are various possible reasons for the changes, including a brutalization of lethal violence or a more effective trauma care. More in-depth analysis of individual cases together with research on victims of attempted homicides is needed to explain these shifts in injury severity.
\end{abstract}

Keywords:

homicide, lethal violence, injury severity score, quantification, longitudinal

\section{INTRODUCTION}

The number of homicides in Sweden has decreased during the last two decades. ${ }^{1}$ In the past 50 years, there was an initial rise in the incidence to a peak level of around 100-120 homicides per year in the 1980s and early 1990s; however, the annual numbers then dropped to around 75 in the early 2010s. Since the population has steadily increased during the same time, the relative incidence has declined even more from the levels of the 1980s. In the early 2010s, the annual homicide incidence was 0.78 per 100 000 inhabitants, in contrast to 1.3 per 100000 in the late 1980s. The reasons for this decline in Sweden as well as in many other West European countries are not known, but possible explanations are lower cultural tolerance for violence, less heavy alcohol drinking, and improvements in emergency care. An exception from the long term decreasing homicide trend in Sweden was seen in 2015, which ended up with 112 homicides, 25 more homicides compared with 2014. Whether this signifies an actual break in trend or a random variation remains to be seen.

Another aspect of interest is the societal response to violent crime. The type of measures taken against offenders differs between places and over time. In Sweden, people who commit violent crimes are often punished with prison sentences, and the harshest sentence is life in prison. A life sentence can be given for different reasons whereof the level of brutality is one. It is notable that there has been a major increase in the number of homicide offenders convicted to life imprisonment in Sweden[1] without any corresponding increase in the rate of homicides. ${ }^{1}$ A possible reason

1 Sturup J., Granath S., Unsolved homicides: The structure of homicides in Sweden from 1990 to 2014, (in review) for the increase in life sentences is a change in the type of penalties with respect to imprisonment vs. psychiatric care. In the late 1980s and early 1990s, a considerably higher proportion of offenders received forensic psychiatric care compared with the present situation.[2] However, this cannot make up for the total increase in life sentenced homicide offenders, and another reason for the increase in life sentences is probably due to an increase in punitive attitude among the general population and likely the courts.[3,4] Yet another reason for the increase in life sentences may be an increase in brutality within the homicides committed, which instigates research regarding possible changes in level of brutalization in homicides. Assessing the brutality of a homicide involves many different parameters: psychological, contextual, and physical. One important parameter in this assessment will surely be the injury severity in the victim. [5]

A useful research tool for analyzing injury severity requires a measure that is valid and reliable. Allen argued that the Abbreviated Injury Scale (AIS) and the Injury Severity Score (ISS), which is based on the AIS, would be valuable tools for measuring injury severity in studies on criminal victimization.[6-8] The strengths of these methods are partly that they are based on objective injury descriptions, which lead to high inter-rater reliability, and partly that they are commonly used and have been validated in trauma research.[9] Their applicability to homicide victims has recently been evaluated together with other injury severity scores.[10] In that study, it was argued that the sum of all AIS scores (SAIS) in a victim could be used as a gold standard for quantifying injuries in homicide victims, since it takes into account both the number of injuries as well as their individual severities in an objective way. The ISS was deemed too crude when compared with this gold standard; while, the homicide injury scale (HIS) was the best alternative when looking for a valid method that was quick and easy to apply.[11] The SAIS is much more time consuming and more medical knowledge is needed to use it. 


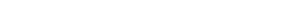

Longitudinal studies of homicides usually include the homicide rate, characteristics of the victim and offender, and the type of violence (blunt force, gunshot, etc.). Few studies have used injury quantification as part of the time trend analysis. In a longitudinal study (1983-1992) of homicides caused by sharp force in the geographical area served by the Department of Forensic Medicine in Stockholm, Sweden, the victims had significantly more injuries during the studied period than the victims from the previous ten-year period.[12] In another study of the same geographical area, the total number of injuries and number of lethal injuries were counted in homicide victims from three time periods; 1976-78, 1986-88, and 1996-98.[13] The number of victims with 40 or more injuries was increased in 1996-98 compared with the first two periods. Furthermore, the proportion of victims with multiple lethal injuries was higher in the last period than in the preceding ones.

The aim of the present study was to further investigate the observations of the latter study [13] by quantifying the degree of injury with the HIS and the number of lethal injuries, and to expand the time period studied to include the years 2006-08. The research question was: Are there significant differences in the injury severity of homicide victims between the different time periods? Another aim was to further improve knowledge of how the HIS behaves in a general homicide population. The HIS was originally defined with elderly female victims in mind which might cause it to behave differently in other populations.

\section{MATERIAL AND METHODS}

\section{1. Materials}

The study had a retrospective design and included all available homicide cases from the Department of Forensic Medicine in Stockholm, Sweden, during the periods 1976-1978, 1986-1988, 1996-1998, and 2006-2008, named below as period 1, 2, 3, and 4, respectively. All cases where the forensic pathologist determined the manner of death as deliberately caused by another person were reviewed for inclusion. A total of 354 cases were reviewed; 96, 98, 84, and 76 cases from period 1, 2, 3, and 4, respectively. Cases in which death had been caused by a secondary trauma, such as a fall from height, hit by vehicle or poisoning $(n=11)$, and cases that were hard to assess due to putrefaction ( $n=20)$, major burning $(n=6)$, long periods of hospital care $(n=9)$, incomplete protocol $(n=4)$, hard to interpret protocols $(n=3)$, and others $(n=5)$ were excluded. The excluded cases $(n=58)$ were divided between the four periods as $10,21,14$, and 13 , respectively.

\subsection{Homicide Injury Scale (HIS)}

The HIS was originally proposed as a means of assessing the degree of injury in elderly female homicide victims.[11] It consists of six levels from 1 to 6 , as defined in Table 1.

By transforming some qualitative definitions of the original HIS into objective measures, the scale can be made less ambiguous and, thus, more reliable.[10] This modified version of the HIS was used in the present study. In the original definition, the term overkill was not explicitly defined. To clarify this point and increase inter-rater reliability, a case was considered overkill if at least one of the following criteria was met:

- Three or more sharp wounds located at the head, neck, or trunk with internal organ injuries (including the pleura and large blood vessels)

- Three or more gunshot wounds located at the head, neck, or trunk with internal organ injuries (including the pleura and large blood vessels)

- A total of 40 or more skin injuries (blunt, sharp, gun-shot)

An injury was considered a cause of death if any of the following was true: - The pathologist that performed the autopsy explicitly assessed the injury as lethal/potentially lethal

- There was an injury to an internal organ (including the pleura and large blood vessel)

- If the pathologist that performed the autopsy assessed that there were signs of mechanical asphyxia, asphyxia was counted as a potential lethal injury

The four most common types of trauma were considered: blunt force, sharp force, gunshot, and asphyxia. Injuries within one modality were counted as one cause of death, regardless of the number of injuries within that modality. For example, one lethal gunshot wound and one lethal knife wound were counted as two causes of death, while two lethal knife wounds were counted as one cause of death.

The HIS assessments for all cases were made by a forensic pathologist (FT), while another forensic pathologist (IT) assessed 50 of the cases for interrater reliability. Each case was assigned a random number using the randomfunction in Microsoft Excel and the cases with the 50 lowest numbers were then selected as the random sample for reliability testing. The test of intrarater reliability (FT) was made with the same 50 cases several months after the original assessments. In addition to an assessment of the HIS score, the age, sex, number of lethal/potentially lethal injuries (as defined above) within each type of trauma (blunt, sharp, gunshot, asphyxia), and the body location of lethal wounds were recorded in each case by one author (FT).

Table 1. Original definition of the Homicide Injury Scale (HIS) adopted from Safarik and Jarvis[11]

\begin{tabular}{cl}
\hline Score & Definition \\
\hline 1 & $\begin{array}{l}\text { Single cause of death only: internal injuries only with no visible related external injuries (e.g., smothering, strangulation, ruptured organs resulting from blunt } \\
\text { force trauma) }\end{array}$ \\
2 & Single cause of death only: internal injuries only with minor related external injuries (e.g., smothered with related abrasions and/or contusions of mouth and face, \\
3 & Strangled with related abrasions or ligature marks) \\
4 & Two or more causes of death: related internal and/or external injuries not identified as either excessive or overkill \\
5 & Single cause of death only: related external injuries identified as either excessive or overkill \\
6 & Two or more causes of death: related internal and/or external injuries in at least one of the causes of death identified as either excessive or overkill
\end{tabular}




\subsection{Analyses}

The results were visualized with graphs using Excel, Statistica and $R$ Commander. Statistical analysis was made in R Commander and Excel. Both the Kruskal-Wallis test (with the Mann-Whitney $U$ as post-hoc test) and Fisher's exact test were used. Bonferroni corrected $p$-values were used for pairwise comparisons. A $p$-value $<0.05$ was considered as statistical significant.

The inter- and intra-rater reliability was assessed using the square weighted Cohen's kappa.

\section{RESULTS}

The total number of included cases was 296, divided between the four periods as $86,77,70$, and 63 , respectively. The characteristics of the included cases are reported in Table 2 . The victims were on average 38 years old and the majority was male.

Table 2. Demographics of 296 homicide victims in Stockholm, Sweden, 1976-78, 1986-88, 1996-98, and 2006-08.

\begin{tabular}{cccc}
\hline & $\mathrm{n}$ & Age (sd) & Percentage male \\
\hline $1976-1978$ & 86 & $36.3(15.6)$ & $65.1 \%$ \\
$1986-1988$ & 77 & $35.9(15.8)$ & $72.7 \%$ \\
$1996-1998$ & 70 & $40.3(15.7)$ & $72.9 \%$ \\
$2006-2008$ & 63 & $41.9(17.3)$ & $68.3 \%$ \\
Total & 296 & $38.3(16.2)$ & $69.6 \%$ \\
\hline
\end{tabular}

As shown in Figure 1, the most dominant HIS scores were HIS 3 and HIS 5 , while HIS 1 did not occur in any of the 296 cases. The proportion of HIS 3 declined from the first to the third time period. Between the third and fourth period the proportion of HIS 3 went back up. Since HIS 3 and 5 are the two dominant groups, HIS 5 naturally showed an inverted development. The
Kruskal-Wallis test with HIS as response variable and time period as grouping variable had a $p$-value of 0.0023 . Pair-wise comparison between the time periods with the Mann-Whitney $U$ test (Bonferroni correction) showed a statistically significant difference between period 1 and 3 .

The different HIS scores can be divided into one group that does not contain overkill in the definition (HIS 1-4) and one group that does (HIS 5-6). As shown in Figure 1, the scores of 3 and 5 were largely dominant. Thus, the time trends for cases with and without overkill (Figure 2) were similar to the time trends of HIS 5 and 3, respectively. The overkill homicides peaked in period 3 and then in period 4 returned to about the level of period 2 . Pairwise comparisons between the time periods using the Fisher's exact test and Bonferroni correction showed statistical significance between period 1 and 3 .

Sharp force was the dominating trauma modality during the study period, but showed a declining tendency in the late part of the study. The proportion of gunshot homicides made an inverted development, with higher numbers in the last two decades compared to the first two. Pairwise comparisons between the periods for each trauma modality were made with the Fisher's exact test and Bonferroni correction. Statistical significance was reached between period 1 and 3 for gunshot, and between period 1 and 2 for asphyxia.

The mean numbers of lethal injuries were 1.9, 2.6, 3.1, and 3.5 in the period 1, 2, 3, and 4, respectively. Thus, there was a steady increase in the mean number of injuries over time, with roughly 0.5 more lethal injuries per time period. The Kruskal-Wallis test with the number of lethal injuries as response variable and time period as grouping variable had a $p$-value of 0.0023 . Pairwise comparison with the Mann-Whitney $U$ test (Bonferroni correction) showed statistically significant differences between period 1 and 3 and period 1 and 4 , respectively.

In a homicide victim there might be more than one type of lethal injury. Such cases will by definition have a HIS equal to 4 or 6 and the lethal injuries will be a mix of different modalities (e.g. blunt and sharp). In cases with HIS 2,3 or 5 , there is solely one type of lethal injury. These single modality cases and their HIS-score distributions are displayed in table 3 and figure 5.

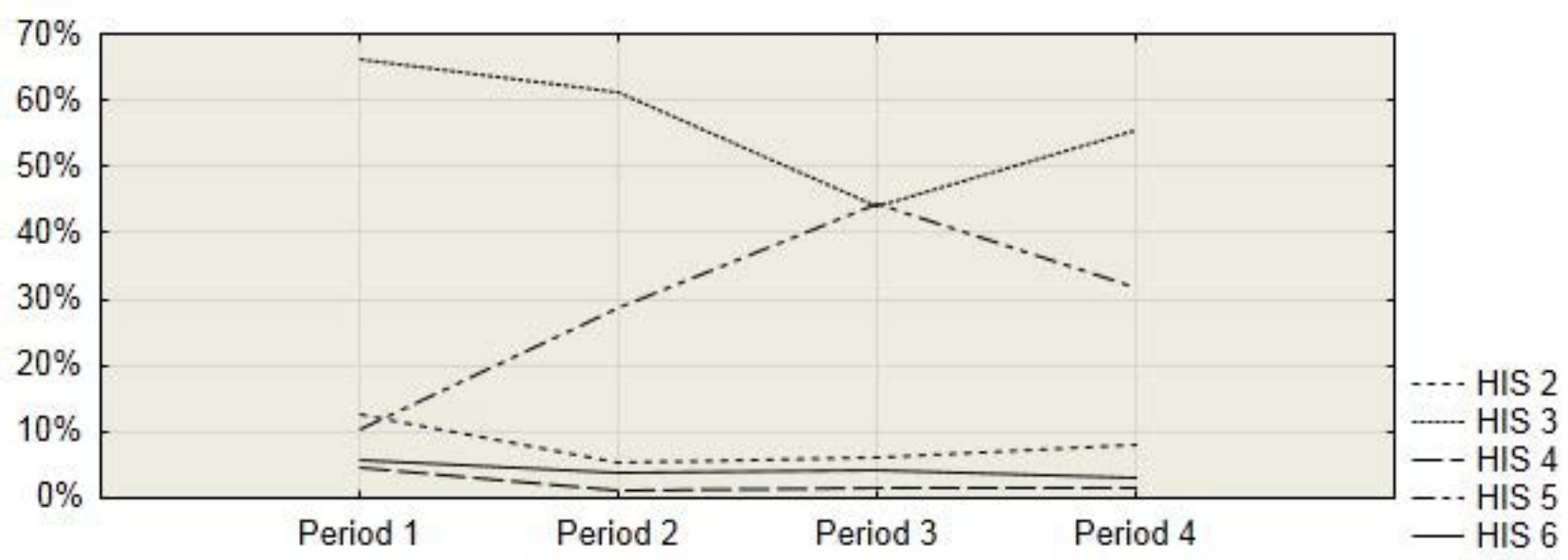

Figure 1 HIS scores. Time trends in relative numbers for HIS scores in 296 homicide victims from the Department of Forensic Medicine in Stockholm during the periods: 1976-1978, 1986-1988, 1996-1998, and 2006-2008. No case with HIS 1 was recorded. 


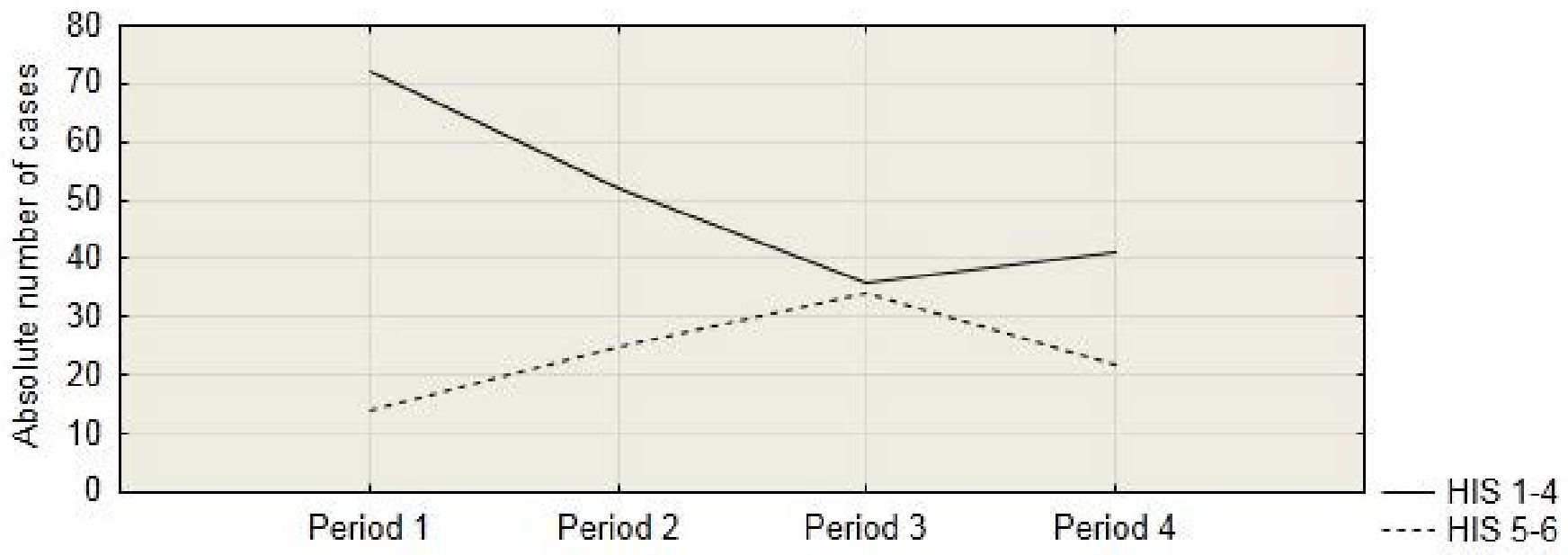

Figure 2. Overkill vs. not overkill. Time trends for HIS scores in absolute numbers, where the scores have been divided into two groups, one without and one with "overkill" in the definition (HIS 1-4 and HIS 5-6, respectively).

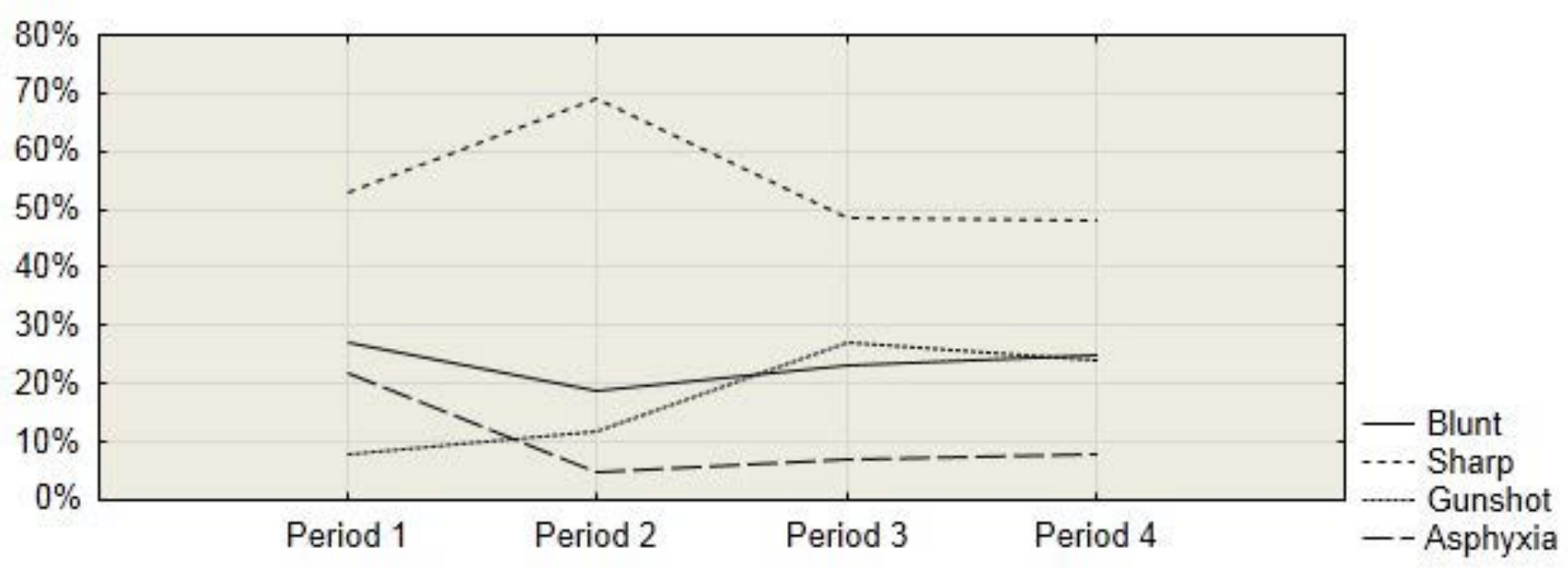

Figure 3. Trauma modalities. Time trends in relative numbers for different trauma modalities. Some victims have lethal injuries in more than one modality, causing the fractions to sum up to more than $100 \%$.

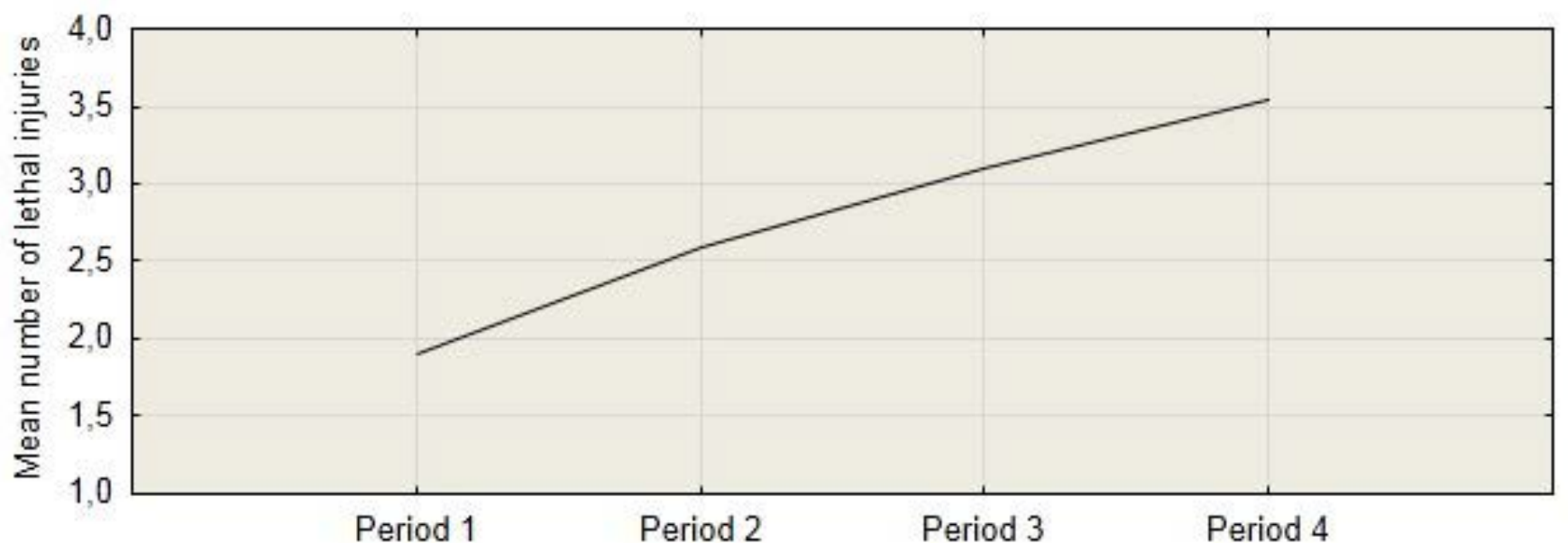

Figure 4. Mean number of lethal injuries per victim. Mean number of lethal injuries in homicide victims from period 1 to period 4. 
Table 3. Proportion of different HIS scores in cases with lethal injuries within only one of the different trauma modalities.

\begin{tabular}{ccccc}
\hline Trauma modality & $\mathrm{n}$ & HIS2 & HIS3 & HIS5 \\
\hline Blunt & 53 & $11 \%$ & $57 \%$ & $32 \%$ \\
Sharp & 149 & $0 \%$ & $65 \%$ & $35 \%$ \\
Gunshot & 49 & $0 \%$ & $78 \%$ & $22 \%$ \\
& & & & \\
Asphyxia & 25 & $72 \%$ & $20 \%$ & $8 \%$ \\
\hline
\end{tabular}

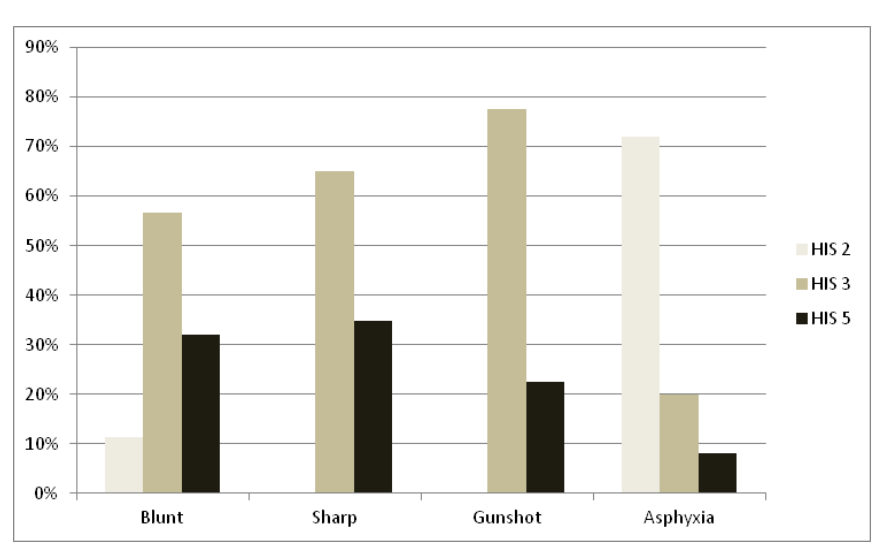

Figure 5. Proportion of different HIS scores in cases with lethal injuries within only one of the different trauma modalities.

The inter- and intra-rater reliability for the HIS using Cohen's kappa (weighted) was 0.74 and 0.95 , respectively. The dominating discrepancy in the interrater reliability test was between HIS 2 and 3, i.e. whether associated injuries were to be assessed as minor vs. moderate to serious.

\section{DISCUSSION}

This population-based study from Stockholm, Sweden showed a trend towards less HIS 3 and more HIS 5 cases from period 1 to period 3, both in relative and absolute numbers. The differences between period 1 and 3 were statistically significant. The increase of HIS 5 cases is consistent with the hypothesis that that there has been a general elevation in the level of aggression exerted by violent offenders, as suggested by Ericsson and Thiblin.[13] Increased aggression would be in line with the increase of life sentences in Sweden. However, it is not possible to draw such a conclusion merely from our findings. First of all, this study concerns the degree of injury displayed by the victims, not the degree of violence or brutality per se. Secondly, there might be alternative explanations other than brutality for changes in injury severity, for example an improvement in trauma care.[14]

With improved trauma care, assault victims that would have died a few decades ago might now survive. It is reasonable to assume that these additionally saved victims on average will have less severe injuries than the ones who still die. This will cause the average homicide victim to have more severe injuries, and thus result in higher injury severity scores on average.

A large study of severe trauma in the San Diego County found a decrease in fatal injuries among the subgroup of assault victims during the period from 1987-1997, while there was no change in the total number of assault victims that were treated. [15] This could for example be explained by improved trauma care or less severe injuries from assault, but the authors do not propose possible explanations for the change. In our material, the mean number of lethal injuries per victim increased monotonically over the time periods, with statistical significance from period 1 to period 3 and 4 , respectively. Possible explanations for this can an improved health care or a shift in the nature of lethal violence. To further investigate such hypothesis, the surviving assault victims must also be studied.

When the HIS scores are divided on trauma modality (table 3 and figure 5), we see that asphyxia differed significantly from the others in that it was much more common with a lower HIS score. This is due to the physiological nature of asphyxia. A person that has been strangled might not have any severe anatomical injuries. A person that has been shot or stabbed will always have external injuries that are classified as moderate to severe, and thus get at least HIS 3 . Thus, the trauma modalities have differences in the nature of their injuries that might need to be considered when comparing groups of homicide victims. The increase of HIS 5 and decrease of HIS 3 between period 1 and 3 might for example be partly explained by a shift in trauma modality, with a statistically significant increase in the proportion of gunshots in period 3 compared to period 1. This might indicate that it would be better to analyze different trauma modalities separately.

Another aspect of the HIS that became apparent in the current study was that the cases are unevenly divided between the six different levels of the scale. The score of 1 was completely absent. We do not find this surprising since there is virtually always some external injury on homicide victims. However, the scores of 2,4 , and 6 were rare as well, and the scale behaved almost dichotomous between 3 and 5 as an indicator of no overkill vs overkill. An objective division of the cases between no overkill and overkill is desirable, but it is a bit too crude. To see changes in injury severity over time or differences between groups, greater variability in a score would be preferable. The HIS was originally proposed for a subgroup of homicide victims, namely elderly females, and this may explain the lack of variation. Since these victims can be expected to be weaker than the average victim, they will not be able to put up great resistance against the attacker. Also, they are more fragile and will be incapacitated by fewer injuries. Both these circumstances will probably result in fewer injuries on average, suggesting the overall injury severity is more due to the brutality of the offender than the resistance of the victim. Thus, the HIS might not be ideal for a general homicide population.

In the original definition of the HIS, "overkill" was said to mean "injury in excess of that that is necessary to cause death". [11] We chose to use a more precise definition of overkill in order to make the HIS more objective and reliable, but the imprecise and emotional nature of the concept of "overkill" might still be confusing. We would therefore suggest that other more neutral terms are used when classifying various degrees of injury. An alternative to "overkill" might be "excessive injuries". Such terms should of course be used together with an explicit definition like the one we used for "overkill" in the HIS. 


\section{METHODOLOGICAL CONSIDERATIONS}

The results of this study indicate that the HIS might be too crude when analyzing a general homicide population. Even though it correlates well with the proposed gold standard (the SAIS) it does not seem to differentiate the cases good enough. This implicates that the HIS can be used to analyze rough trends on a group level, but that it lacks the ability to detect smaller nuances in injury severity.

A limitation of using injury severity scores to measure the injuries in homicide victims is validity. It is obvious that a single number cannot replace the complex, subjective assessment of injury severity. However, as we argued in an earlier study, we can achieve validity on a group level. [10] As long as we do not use injury severity scores to draw far-reaching conclusions in individual cases, they can serve as a valuable tool in homicide research.

Because of a lack of digital archive for the first two studied time periods, and a suboptimal digital archive for the third, it was not straightforward to identify all cases to review for inclusion. Great efforts were made to find all cases, but some may have been missed. The potentially missed cases are deemed to be small in number and randomly selected. Therefore, we believe that this problem has only minor, if any, effects on the results.

Since the years included in the study only comprises $30 \%$ of each decade, the results cannot be directly interpreted as the actual time trend from the 1970s to the 2000s. Before drawing conclusions on the overall development of injury severity from the 1970s to the 2000s, one has to do a study using a random sample from all years in each decade.

The present study consists of cases from only one large city and its surroundings. Therefore, it might be that some aspects are less valid for small towns or rural areas in Sweden. For instance, shootings in criminal settlements are likely to be overrepresented in the type of area studied here. As seen in figure 5, gunshot deaths have a lower proportion of HIS 5 than deaths from both sharp and blunt injuries. Thus, a population from a large city area where gunshot deaths are overrepresented might have a lower proportion of overkill according to the HIS than populations from rural areas where deaths from sharp and blunt injuries might be more common.

One possible limitation in a study based on autopsy protocols is that individual protocol styles differ between different forensic pathologists, but as long as the protocols are relatively homogenous in their structure (which they were in the present study) we argue that this at most has influenced the results in a minor way.

\section{CONCLUSIONS}

The present study indicates that according to the HIS there was an increase in the injury severity of lethal violence in the Stockholm area from the years 1976-78 to 1996-98. The average number of lethal injuries per victim was significantly higher in both 1996-98 and 2006- 2008 compared to 197678. The current study did not aim to explain these changes, but future studies might look into different hypothesis such as a brutalization of lethal violence, or a more effective trauma care. Finally, the HIS was developed for quantifying injury severity in elderly female victims. The results of the current study raise the question of whether the (modified) HIS is to crude to be used in general homicide cases due to different injury patterns.

\section{ACKNOWLEDGEMENTS}

The study was financially supported by the Swedish National Board of Forensic Medicine.

\section{REFERENCES}

[1] Sturup J., Karlberg D., Fredriksson B., Lihoff T., Kristiansson M., Risk assessments and recidivism among a population-based group of Swedish offenders sentenced to life in prison, Crim Behav Ment Health, 2016, 26, 124-135

[2] Sturup J., Lindqvist P., Homicide and psychosis 1987-2006 - A Swedish time trend analysis, International Journal of Forensic Mental Health, 2014, 1-7

[3] Granath S. (2007) The justice System's response to youth crime 1980-2005. Stockholm University, Stockholm

[4] Jerre K., Tham H. (2010) The Swedes' view on punishment (Svenskarnas syn på straff). Institution of Criminology, Stockholm

[5] Douglas J.E., Burgess A.W., Burgess A.G., Ressler R.K. (eds) (2006) Crime Classification Manual: A standard system for investigating and classifying violent crimes. 2 edn. Jossey-Bass, San Fransisco

[6] Allen R., Measuring the Severity of Physical Injury Among Assault and Homicide Victims, Journal of Quantitative Criminology, 1986, 2, 139-156

[7] Gennarelli T.A., Wodzin E. (eds) (2008) Abbreviated Injury Scale 2005 - Update 2008. Association for the Advancement of Automotive Medicine, Barrington

[8] Baker S.P., O'Neill B., Haddon W., Jr., Long W.B., The injury severity score: a method for describing patients with multiple injuries and evaluating emergency care, J Trauma, $1974,14,187-196$
[9] Tohira H., Jacobs I., Mountain D., Gibson N., Yeo A., Systematic review of predictive performance of injury severity scoring tools, Scandinavian Journal of Trauma Resuscitation \& Emergency Medicine, 2012, 20,

[10] Tamsen F., Klötz-Logan F., Thiblin I., Homicide Injury Quantification: Correlations and Reliability of Injury Severity Scores Applied to Homicide Victims, Homicide Studies, $2015,19,88-100$

[11] Safarik M.E., Jarvis J.R., Examining attributes of homicides - Toward quantifying qualitative values of injury severity, Homicide Studies, 2005, 9, 183-203

[12] Karlsson T., Sharp force homicides in the Stockholm area, 1983-1992, Forensic Science International, 1998, 94, 129-139

[13] Ericsson A., Thiblin I., Injuries inflicted on homicide victims. A longitudinal victiminologic study of lethal violence, Forensic Sci Int, 2002, 130, 133-139

[14] Harris A.R., Thomas S.H., Fischer G.A., Hirsch D.J., Murder and Medicine. The Lethality of Criminal Assault 1960-1999., Homicide Studies, 2002, 6, 128-166

[15] Potenza B.M., Hoyt D.B., Coimbra R., Fortlage D., Holbrook T., Hollingsworth-Fridlund P., Trauma Res Educ F., The epidemiology of serious and fatal injury in San Diego county over an 11-year period, Journal of Trauma-Injury Infection and Critical Care, $2004,56,68-75$ 